\title{
The Latent Profiles of Life Domain Importance and Satisfaction in a Quality of Life Scale
}

\author{
Ssu-Kuang Chen • Sunny S. J. Lin
}

Accepted: 11 March 2013/Published online: 21 March 2013

(C) Springer Science+Business Media Dordrecht 2013

\begin{abstract}
The present study aimed to use a latent profile analysis to distinguish between populations in terms of life domain importance and satisfaction profiles. Then, a multinomial logistic regression was used to determine how background variables (e.g., gender, living areas, and school levels) and self-perceived health predict each latent quality of life (QoL) profile. We also investigated how the latent groups of adolescents predicted negative and positive wellbeing indicators (e.g., problem behaviors and overall life satisfaction). The sample consisted of 720 Taiwanese secondary school students. Three latent groups were established as follows: "unimportant-unsatisfied," "important-unsatisfied," and "important-satisfied." The results indicate the following: (a) boys were more likely to fall into the "unimportant-unsatisfied" group than were girls; (b) better health increased the likelihood of being in the "importantsatisfied" group; (c) high school students were more likely to be in the "unimportant-unsatisfed" group than were middle school students; and (d) no relationship was found between latent groups and living areas. The function of importance rating was not present when evaluating the importance-satisfaction profiles and their relationship with problem behaviors and overall life satisfaction. The problems of the "unimportant-unsatisfied" profile among youth are discussed.
\end{abstract}

Keywords Quality of life · Life domain importance - Life satisfaction ·

Latent profile model

\section{Introduction}

\subsection{Importance and Satisfaction in Quality of Life Research}

Including subjective assessments of importance in measurements of quality of life (QoL) has had intuitive appeal and has drawn attention from researchers in this area (Hsieh 2008,

S.-K. Chen · S. S. J. Lin $(\bowtie)$

Institute of Education, National Chiao Tung University, 1001 Ta-Hsueh Rd, Hsinchu 300, Taiwan R.O.C

e-mail: sunnylin.nctu@gmail.com

S.-K. Chen

e-mail: Schen75025@gmail.com 
2012a; Meuleners and Lee 2005; Raphael et al. 1996; Russell et al. 2006; Trauer and Mackinnon 2001; Wallander et al. 2001; Wu et al. 2009). For this type of assessment, respondents are asked to rate the importance of and their satisfaction with various life domains. These life domains include physical health, living environment, school, job, etc. Subjective importance indicates how important individuals perceive each life domain to be. Importance ratings offer valuable information because certain life domains might be critical to one individual but not to others. Understanding the subjective importance of each domain allows health practitioners, educators, and the community to provide efficient help for improving QoL. Researchers have studied the possibility of incorporating importance weightings into satisfaction scores to derive an overall QoL index (Hsieh 2003; Russell et al. 2006). However, there is mixed empirical evidence on whether the calculation of an overall QoL index should take into account subjective importance and how importance ratings should be used together with satisfaction ratings to more accurately reflect overall QoL.

Among a variety of calculation mechanisms for an overall QoL measure, Hsieh (2003) assumed ratings and rankings functioned differently in the calculations and found that importance rankings improved the correlation between the single-item global satisfaction measure and domain satisfaction scores. However, Wu's result (2008a) revealed that weighted satisfaction scores are not superior to unweighted satisfaction scores in predicting overall life satisfaction. The theory behind this finding claims that an individual's rating of domain satisfaction essentially depends on whether the domain is important or not; importance has already been considered spontaneously when evaluating domain satisfaction, so that adding the weighting into an overall QoL measure would be redundant (Trauer and Mackinnon 2001; Wu and Yao 2006a, 2007a; Wu 2008a, 2008b). Trauer and Mackinnon (2001) argued that an individual is unlikely to feel strongly about a domain unless that domain is considered important to him/her (also see Russell and Hubley 2005; $\mathrm{Wu}$ and Yao 2006a). The finding supporting this argument includes the result that the range of satisfaction scores is maximized only when the domain is considered important (Trauer and Mackinnon 2001). Locke's range-of-affect hypothesis (Locke 1969) in job satisfaction theory was borrowed to demonstrate that how satisfaction is formed depends on the perceived importance of the domain (Wu and Yao 2006a; Wu 2008a, 2008b). According to Locke's range-of-affect hypothesis (Locke 1969), job satisfaction would be greatest when the discrepancy of have-want is small for the domains that are important; job dissatisfaction would be smallest when the discrepancy of have-want is large for the domains that are important. Domains with low personal importance are less likely to generate strong affective reactions, either negative or positive.

Because satisfaction judgments may involve importance evaluations, the question of whether importance and satisfaction ratings measure the same construct has arisen. So far, no one has examined the latent constructs of importance and satisfaction using factor analysis (Russell and Hubley 2005); however, it is agreed that importance and satisfaction are two different constructs, based on the finding of a lack of relationship between importance and satisfaction ratings. For example, Wu and Yao (2006b) found that domain importance weightings were not related to domain satisfaction for 12 out of 15 domains. $\mathrm{Wu}(2008 \mathrm{a}, 2008 \mathrm{~b})$ reported that the correlations between satisfaction and importance were not consistent for different domains: some were significantly positive, some were significantly negative, and others were not significant. Russell et al. (2006) reported correlations ranging from -.13 to .31 for importance and satisfaction ratings across 21 domains of a QoL measure for injection drug users. Trauer and Mackinnon (2001) also showed that extreme dissatisfaction or satisfaction ratings were associated with higher importance ratings, implying no linear relationship between them. 
To further understand whether importance is spontaneously incorporated into satisfaction judgments, the role of importance in the prediction of overall satisfaction from domain satisfaction has been investigated. In Wu and Yao's (2006a, 2006b) studies, the external criterion, the Satisfaction with Life Scale (SWLS; Diener et al. 1985) score, was regressed on domain satisfaction, importance, and the interaction of satisfaction and importance for each domain. In general, there was no moderating effect of importance; moderator effects were found only for a few of the domains ( $\mathrm{Wu}$ and Yao 2006a). However, using a different research approach, Hsieh (2012a) conducted a z-test on the correlation between the sum of domain satisfaction scores and overall satisfaction ratings for different clusters of people, who were grouped by importance ranking profiles for various life domains. The relationship between overall life satisfaction and the sum of domain satisfaction scores was not the same for respondents with different importance ranking profiles, suggesting an importance effect of domain in QoL measures.

Based on the previous empirical evidence, most researchers object to using a weighting mechanism to combine importance weightings and satisfaction ratings in an overall QoL measure. However, Hsieh (2012b, 2011) noted that importance weighting has its own value when there are a sufficient number of different domains in a satisfaction scale. Importance weightings take into account the potential individual differences in the perceived importance of various life domains; this is more obvious when more life domains are covered in the survey. Moreover, researchers should reevaluate the limitations of previous studies; for example, domain importance evaluated with a scale may not be applied to the other QoL scales or samples (Wu and Yao 2006a). In the past, the literature has shown that high or low domain satisfaction reflects high importance of the domain in respondents' minds, and people who consider a domain unimportant generally give a neutral satisfaction rating (Wu and Yao 2006a). Could it be that people who report feeling unsatisfied in a particular domain consider this domain unimportant? Feeling unsatisfied with a domain has a different meaning for people who also rate this domain as unimportant compared to those who consider the domain to be important.

\subsection{The Present Study}

Most researchers agree that the most practical function of importance rating is for item selection or QoL measurement development. Beyond this, from the perspective of research on individual differences in human development, we believe that evaluating perceived importance will enhance the understanding of the perceptions or attitudes towards life domains for different populations. The present study took this perspective with an adolescent target population, as more and more research attention has been paid to this particular life stage. In the past, different statistical analyses have been applied to investigate the relationships between satisfaction ratings, importance ratings, and external criteria (e.g., overall life satisfaction), but have mostly relied on a variable-centered approach, using correlation, regression, etc. The person-centered approach has only been considered in examining different types of people with similar importance ranking patterns (Hsieh 2012a). To our knowledge, no one has yet examined the profiles of importance and satisfaction simultaneously using a person-centered approach. It has not yet been discussed which are the latent groups that show different profiles of importance and satisfaction ratings. By performing latent profile analyses, people are classified so that all individuals in a group have similar profiles. Identifying latent profiles and classifying respondents according to their individual importance and satisfaction profiles is likely to provide insights into individual differences in QoL research (Magnusson and Cairns 1996) and 
support investigations of the relationships between domain importance and satisfaction. In sum, the present study aimed to examine what types of adolescents would show similar rating profiles of importance and satisfaction in a QoL scale. The particular research questions were as follows:

(1) What are the latent types of adolescents who perceive importance and satisfaction similarly for different life domains?

(2) How does each latent group of adolescent differ in other variables/covariates, such as background (e.g., gender, living areas, school levels), self-perceived health, and negative and positive well-being indicators (e.g., problem behaviors and overall life satisfaction)?

\subsection{Latent Profile Analysis}

Latent profile models can be used to identify latent and homogeneous populations within large heterogeneous populations, with meaningful groups of individuals profiled in terms of observable variables (for a detailed overview, see Magidson and Vermunt 2004 and Muthén 2001). In studies of learning, latent profile analyses of achievement goal orientations have resulted in better differentiation and more accurate predictions of subjective well-being (Tuominen-Soini et al. 2008). Furthermore, in psychiatric studies, latent profile analyses have helped in the identification of depressed adolescent subtypes, thus providing usable data in support of optimum treatment and prevention decisions (Herman et al. 2007).

The basic model of latent profile has the form $f\left(y_{i} \mid \theta\right)=\sum_{k=1}^{k} \pi_{k} f_{k}\left(y_{i} \mid \boldsymbol{\mu}_{\mathbf{k}}, \boldsymbol{\Sigma}_{\mathbf{k}}\right)$, where $y_{i}$ denotes a vector of indicators of cluster membership for person $i$ in the sample of size $N$ $(i=1, \ldots, N)$. The above probability function (or distribution) represents the probability of a pattern of indicator scores given $\theta\left(\theta=\pi_{1}, \mu_{1}, \sigma_{1}^{2}, \pi_{2}, \mu_{2}, \sigma_{2}^{2}, \ldots, \pi_{k}, \mu_{k}, \sigma_{k}^{2}\right)$, where $k$ is the number of clusters. The distribution for each cluster $k$ is defined by a mean vector $\boldsymbol{\mu}_{\mathbf{k}}$ and the covariance matrix $\boldsymbol{\Sigma}_{\boldsymbol{\kappa}}, \pi_{k}$ represents the weights of each probability function (or prior probability of belonging to the latent class $k$ ). The sum of weights for each cluster is 1.00. The main objective of latent profile analysis is to find classes that differ with respect to their means or locations.

\section{Method}

\subsection{Sample}

The present study was part of a national adolescent well-being survey in Taiwan, in which the data were collected with gratified sampling. The participants were from secondary schools selected from each regional cluster as classified according to the official Taiwan territorial divisions (northwest, midwest, southwest, and east/islands). The numbers and percentages of participating schools and students in each region were determined based on the educational statistics published by the Taiwan Ministry of Education. There were 602 middle school students (297 males, 305 females) and 790 high school students (457 males, 333 females) in the final sample. Informed consent was obtained, and the participants were asked to complete questions on their backgrounds, the perceived importance of and satisfaction with a variety of life domains, their overall life satisfaction, and problem behaviors. 


\subsection{Instrument}

The Quality of Life Profile Adolescent Version (QOLPAV; Raphael et al. 1996) was used in the present study. According to Wallander et al's (2001) recommendation, the QOLPAV is one of five generic adolescent QoL scales worthy for detailed study. The QOLPAV was developed based on a Centre for Health Promotion Model (CHP; Raphael et al. 1996) and includes 54 items that examine three major QoL domains of adolescence: being, belonging, and becoming. Each domain contains three sub-domains and each sub-domain contains three items. The descriptions of the sub-domains are as follows:

(1) Physical Being: Physical health, mobility, nutrition, fitness, and appearance;

(2) Psychological Being: Independence, autonomy, self-acceptance, and freedom from stress;

(3) Spiritual Being: Personal values and standards, and spiritual beliefs;

(4) Physical Belonging: Physical aspects of the immediate environment;

(5) Social Belonging: Relationships with family, friends, and acquaintances;

(6) Community Belonging: Availability of societal resources and services;

(7) Practical Becoming: Home, school and work activities;

(8) Leisure Becoming: Indoor and outdoor activities, recreational resources; and

(9) Growth Becoming: Learning things, improving skills and relationships, and adapting.

We forward translated the QOLPAV scale into Chinese. Backward translation was conducted by a translator who had never seen the original survey questions. The backward translation was checked against the original to ensure there were no discrepancies between the versions. The participants rated the importance of and their satisfaction with each item using a 5-point Likert scale.

The measurement properties of the QOLPAV have been examined in terms of factor structures, convergent validity, and item response model based item analysis (Chen et al. 2011; Bradford et al. 2002; Meuleners and Lee 2005; Meuleners et al. 2003; Raphael et al. 1996). Chen et al. (2011) found that some of the items were poor in certain psychometric characteristics (e.g., item discrimination); therefore, not all of the 54 items were used in the following analysis. We used a short version based on the adequacy of item contents and included the items found in previous research to have higher factor loadings (Chen et al. 2011). In the short version, each factor only contained three items, for a total of 27 items tapping importance and satisfaction.

The covariates of interest in the present study included gender, school level, living area, self-perceived health, overall life satisfaction, and problem behaviors. School levels were split into middle school and high school. Living area was coded as either urban or nonurban. Self-perceived health was rated using 5-point Likert ratings from 1 ("very bad") to 5 ("very good"). The Satisfaction with Life Scale (Diener et al. 1985) with five items was used to evaluate overall life satisfaction. We used 12 problem behavior questions to ask how frequently students displayed problem behaviors, such as skipping school without a legitimate excuse, exhibiting violent behaviors, etc. A score of 1 represents "never" and 5 refers to "10 times or more." The higher the average score, the more frequent the occurrence of problem behaviors is.

\subsection{Data Analysis}

Data analysis was performed using Mplus 6 (Muthén and Muthén 2010) and included three steps. First, confirmatory factor analysis (CFA) was conducted to evaluate the latent factor 
model. Second, the model was cross-validated using CFA with an independent sample. Third, the latent profiles were analyzed to identify the latent groups. To conduct the above analyses, two non-overlapping groups from the sample were randomly selected. Sample 1 $(N=672)$ was used to perform the CFA and evaluate the model-data fit and modification indices. Sample $2(N=720)$ was used to conduct a separate CFA to cross-validate the final factor structure suggested by the first analysis. The factor scores generated for Sample 2 were used as the input data in the latent profile analysis.

\subsubsection{Confirmatory Factor Analysis}

Although the design of the QOLPAV indicates three higher-order factors (i.e., being, belonging, and becoming), to investigate the latent profile based on a variety of QoL domains an 18-factor model (nine for importance and nine for satisfaction) without higherorder factors was adopted. In the model, the correlations between factors were free to be estimated and the error terms of the importance and satisfaction ratings for the same item were allowed to be correlated.

\subsubsection{Latent Profile Analysis}

Multiple latent profile models (2-group to 4-group) were specified and evaluated to find the best-fitting model and the most appropriate number of latent groups. The statistics that are most often used to select the best-fitting model include Akaike's (1987) Information Criterion (AIC), Schwartz's (1978) Bayesian Information Criterion (BIC), and Sclove's (1987) Adjusted BIC (ABIC). Lower values indicate better model-data fit and a more parsimonious model given two models with similar fit to the same data. When comparing models of $k$ and $k-1$ groups, the Vuong-Lo-Mendell-Rubin likelihood ratio test (VLMR) (Lo et al. 2001) produces $p$ values that indicate whether the $k$-group model fits significantly better than the $k$-1-group model. Mplus calculated the probabilities of membership in each group for each individual and assigned individuals to the group associated with the highest probability. Precision-of-classification indicators (e.g., entropy statistics) were adopted to assess the classification utility of the model. By consulting the above statistics, the final model was determined.

\section{Results}

\subsection{Descriptive Statistics on the Samples}

Table 1 shows background information for Samples 1 and 2. Samples 1 and 2 were similar in terms of distribution by gender, school level, and living area. Independent $t$-tests were performed to examine whether Sample 1 and Sample 2 differed in self-perceived health, overall life satisfaction, and problem behaviors. No sample differences were noted for overall life satisfaction and problem behaviors. The test results showed that Sample 1 exhibited slightly better self-perceived health than did Sample $2(M=2.790, S D=1.081$, $N=657$ vs. $M=2.670, S D=1.018, N=702 ; t[1337.603]=2.026, p<.05)$. Cohen's $d$ value for the $t$-test for self-perceived health was small (.110). In general, the two samples consisted of students with similar backgrounds and other measurements. 
Table 1 Sample background

\begin{tabular}{lllll}
\hline & & & $\mathrm{N}$ & $\%$ \\
\hline Sample 1 & \multirow{2}{*}{ Gender } & Boys & 364 & 54.20 \\
& & Girls & 308 & 45.80 \\
& Level & Middle & 310 & 46.10 \\
& & High & 362 & 53.90 \\
& Area & Urban & 393 & 58.50 \\
Sample 2 & \multirow{2}{*}{ Gender } & Others & 279 & 41.50 \\
& & Boys & 390 & 54.20 \\
& \multirow{2}{*}{ Level } & Girls & 330 & 45.80 \\
& & Middle & 292 & 40.60 \\
& Area & High & 428 & 59.40 \\
& & Urban & 396 & 55.00 \\
& & Others & 324 & 45.00 \\
\hline
\end{tabular}

\subsection{Confirmatory Factor Analysis}

The 18-factor model was tested and showed a good fit to the Sample 1 data $\left(\chi_{(1197)}^{2}=2165.351, p<.001 ; \mathrm{CFI}=.946 ; \mathrm{RMSEA}=.035 ; \mathrm{SRMR}=.042\right) . \mathrm{We}$ crossvalidated this model with Sample 2 and found an adequate level of fit $\left(\chi_{(1197)}^{2}=2426.213\right.$, $p<.001$; CFI $=.931$; RMSEA $=.038$; SRMR $=.048)$. The Cronbach's alpha for each factor was at least .60 .

\subsection{Latent Profiles}

As shown in Table 2, AIC, BIC, and ABIC decreased slightly after the fourth group was specified, indicating little improvement in the 4-group latent profile model. The $p$ value from the VLMR likelihood ratio test ( $p$-VLMR) suggested no statistically significant difference between the 4-group model and the 3-group model; that is, the 4-group model did not improve model specification compared to the 3-group model. According to the entropy value for the 3-group model (.927), a high level of precision in assigning individuals to their appropriate groups was found. Therefore, the 3-group model was considered to be the best-fitting model.

Figure 1 displays the latent profiles with nine importance and nine satisfaction indicators for the three latent groups. According to their profiles (Fig. 1), Group 1 students were students who perceived most of the life domains as unimportant and were unsatisfied with them $(21.30 \%)$; Group 2 students were those who considered most of the life domains slightly more important but were also unsatisfied with them (38.60\%); and Group 3 had slightly higher importance ratings for the life domains and were satisfied with their lives in general $(40.11 \%)$.

Therefore, the three groups were labeled as "unimportant-unsatisfied," "importantunsatisfied," and "important-satisfied" to reflect the perceptions of the nine life domains of Groups 1, 2, and 3, respectively. To examine group differences based on the profile indicators, one-way ANOVA analyses and post hoc comparisons were performed for the nine importance indicators and the nine satisfaction indicators individually. The results showed statistically significant differences in average importance indicators (i.e., factor 
Table 2 Latent profile model fit statistics

\begin{tabular}{llllllll}
\hline $\begin{array}{l}\text { Number } \\
\text { of groups }\end{array}$ & $d f$ & Log likelihood & BIC & ABIC & AIC & $p$-VLMR & Entropy \\
\hline 2 & 55 & -7755.282 & 15872.424 & 15697.783 & 15620.565 & 0.000 & 0.957 \\
3 & 74 & -6786.235 & 14059.334 & 13824.364 & 13720.470 & 0.004 & 0.927 \\
4 & 93 & -6291.586 & 13195.043 & 12899.742 & 12769.173 & 0.306 & 0.926 \\
\hline
\end{tabular}

Fig. 1 Latent profiles of importance and satisfaction factors. In the $X$-axis, letters $a$ and $b$ represent the importance and satisfaction factors, respectively. Numbers 1 through 9 are the factor numbers. For example, $6 b$ is Satisfaction Factor 6

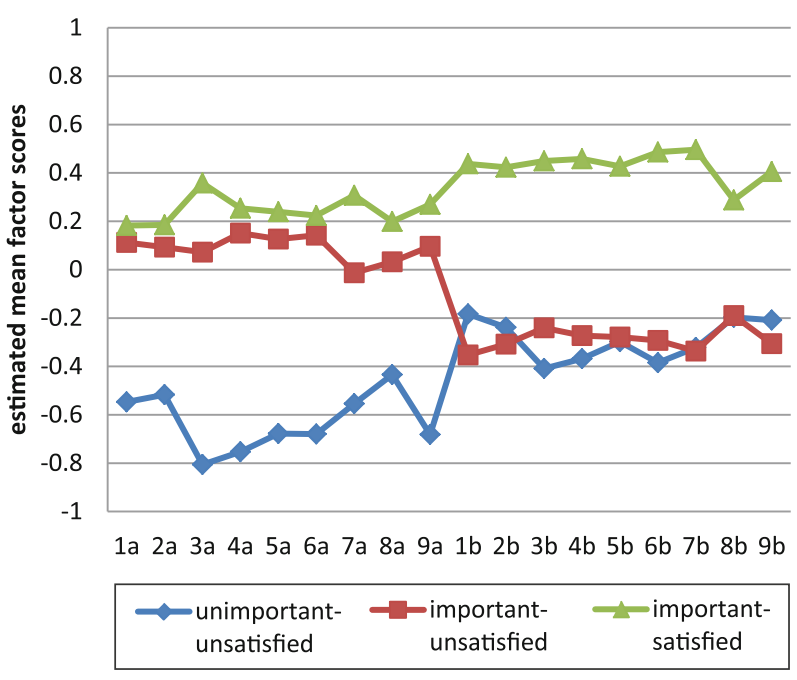

scores) among the three groups $(f[2,717]$ ranging from 246.02 to 542.143 , ps $<.001$, partial $\eta^{2}$ ranged from .407 to .602). The Games-Howell test results indicated that all the importance indicators were different for each group. As for the satisfaction indicators, almost all the satisfaction indicators exhibited similar values between the "unimportantunsatisfied" and "important-unsatisfied" groups. The exceptions were Satisfaction Factors 1,3 , and 9 between the "unimportant-unsatisfied" and the "important-unsatisfied" groups (Satisfaction Factor $1: f[2,717]=189.608, p<.001$, partial $\eta^{2}=.346$, Games-Howell test $p<.05$; Satisfaction Factor 3: $f[2,717]=276.788, p<.001$, partial $\eta^{2}=.436$, Games-Howell test $p<.05$; Satisfaction Factor 9: $f[2,717]=265.179, p<.001$, partial $\eta^{2}=.425$, Games-Howell test $p<.05$ ). These results provided evidence that the latent groups were different in importance and satisfaction indicators in general. The profile of Group 1 ("unimportant-unsatisfied") was examined with particular interest because this group might require extra attention from adolescence researchers. These students were low in their importance ratings of Factor 3, "my beliefs and values," but scored high on Factor 8, “things I do for enjoyment."

\subsection{Latent Groups and Covariates}

In terms of measurement quality, the problem behaviors scale had median to high internal consistency (Sample 2: $N=713$, Cronbach's alpha $=.756$ ). The Satisfaction with Life Scale (Diener et al. 1985) was a scale with high internal consistency (Sample 2: $N=711$, Cronbach's alpha $=.805)$. 
Table 3 Multinomial logistic regression coefficients and odds ratios for the three groups using gender, level, living area, and health rating as predictors ("important-satisfied" = reference group)

\begin{tabular}{llcrrr}
\hline Group & Effect & \multicolumn{1}{l}{$\beta$} & SE & Wald's $\chi^{2}$ & Odds ratio \\
\hline Unimportant-unsatisfied & Intercept & 0.529 & 0.358 & 2.183 & \\
& Gender (female) & $-0.535^{* * *}$ & 0.215 & 6.204 & 0.586 \\
& Level (high school) & $0.693^{* * *}$ & 0.216 & 10.297 & 2.000 \\
& Health & $-0.447^{* * *}$ & 0.108 & 17.309 & 0.639 \\
& Area (urban) & -0.286 & 0.214 & 1.775 & 0.752 \\
Important-unsatisfied & Intercept & 0.258 & 0.321 & 0.647 & \\
& Gender (female) & 0.077 & 0.181 & 0.182 & 1.080 \\
& Level (high school) & $1.307^{* * *}$ & 0.191 & 46.826 & 3.694 \\
& Health & $-0.385^{* * *}$ & 0.091 & 17.798 & 0.680 \\
& Area (urban) & -0.194 & 0.184 & 1.118 & 0.824 \\
\hline
\end{tabular}

Cox and Snell $R^{2}=0.128$, Nagelkerke $R^{2}=0.145$, McFadden $R^{2}=0.064$

$* * p<.01, * * * p<.001$

To investigate the relationship between the latent groups and covariates, a multinomial logistic regression analysis for Sample 2 was conducted using gender, school level, living area, and self-perceived health as predictors. The results indicated that compared to the "important-satisfied" group (i.e., reference group), boys had a higher probability of being in the "unimportant-unsatisfied" group. Healthier students had a lower probability of being in the "unimportant-unsatisfied" and "important-unsatisfied" groups. High school students had a higher probability of being in the "important-unsatisfied" or the "unimportantunsatisfied" group rather than the "important-satisfied" group. Living area did not evidence different probabilities among the three groups (see Table 3).

As for the relationships between the latent groups and overall life satisfaction scores, a one-way ANOVA was conducted and revealed that overall life satisfaction was different between the "unimportant-unsatisfied" and "important-satisfied" groups and between the "important-unsatisfied" and "important-satisfied" groups (see Table 4, Games-Howell test ps <.001). There was no difference, however, in overall life satisfaction between the "important-unsatisfied" and "unimportant-unsatisfied" groups (Games-Howell test $p=.312)$.

In an ANOVA using problem behaviors as the outcome, we found a group difference (see Table 4) and post hoc differences between the "unimportant-unsatisfied" and "important-satisfied" groups and between the "important-unsatisfied" and "importantsatisfied" groups (Games-Howell test for the former, $p<.005$ and for the latter, $p<.05$ ). The results showed no significant difference between the "important-unsatisfied" and "unimportant-unsatisfied" groups (Games-Howell test $p=.543$ ).

In order to further investigate whether the group difference still exists after controlling gender, school level, living area, and self-perceived health, an ANCOVA was performed in which the group of life importance and satisfaction profile, four covariates, and all possible interactions were included. Because the interactions were not significant, only main effects were considered in the second run of the ANCOVA. The results (see Table 5) indicated that after four covariates were held constant the three groups still differed in overall life satisfaction. The pairwise comparisons of estimated marginal means for the three groups (see Table 4) revealed that there were differences between the "unimportant-unsatisfied" and "important-satisfied" groups $(3.33$ vs. $4.20, p<.001)$ and between the "important- 
unsatisfied" and "important-satisfied" groups (3.51 vs. 4.20, $p<.001)$; however, no difference was found between the "important-unsatisfied" and "unimportant-unsatisfied" groups ( 3.51 vs. $3.33, p=.12$ ). Similarly, the three groups also showed significantly main effect in problem behaviors after controlling the four covariates. It should be noted that gender was the most dominant predictor of problem behaviors. A pairwise comparison result similar to that of overall life satisfaction was obtained.

\section{Discussion}

\subsection{Latent Profile Results}

The first research question addressed in the present study examined the types of adolescents that best describe the profiles of perceived importance of and satisfaction with various life domains. A latent profile analysis was performed and identified three types of adolescents: "important-satisfied," "important-unsatisfied," and "unimportant-unsatisfied." The first two groups were the adolescents who regarded almost all of the life domains as important (above average and relatively higher than the third group). Some of these adolescents felt satisfied with the life domains (e.g., "important-satisfied") but others did not (e.g., "important-unsatisfied"). The third group ("unimportant-unsatisfied"), comprised of approximately $21 \%$ of the adolescents in our sample, did not rate the life domains measuring QoL as being as important as did the other groups.

Trauer and Mackinnon (2001) and Locke (1969) suggested there may be three types of importance and satisfaction ratings: "important-satisfied," "important-unsatisfied," and "unimportant-neutral." In contrast to their findings with adults, our results exhibited a potential profile of "unimportant-unsatisfied" adolescents. The existence of this group of adolescents does not support the range-of-affect hypothesis in job/life satisfaction that assumes people who consider themselves satisfied or unsatisfied in a domain have intuitively regarded the domain as important. Special attention must be paid to the "unimportant-unsatisfied" group by QoL researchers. Putting the argument about importance weighting aside, if we consider "domain importance" redundant and only include satisfaction when studying adolescents' QoL, we may miss some crucial information about adolescents' perceptions of various life aspects, or the attitudes behind their evaluations of QoL. If a QoL index was proposed to evaluate QoL and ultimately aimed to improve the QoL of adolescents, the importance ratings of life domains would provide additional information that should not be neglected in the research on adolescents' well-being.

Among all the unimportant life domains for the "unimportant-unsatisfied" group, the domain rated slightly more important than the others was "things I do for enjoyment," while the least important was "beliefs and values" (e.g., having hope for the future; feeling that life has meaning). This group of adolescents felt unsatisfied with life in general. Though the findings are limited, the role of meaning in life in relation to an individual's perceived QoL has been explored with adolescents (Ho et al. 2010) and results suggest that both meaning in life and optimism are positively associated with life satisfaction. The profile of the "unimportant-unsatisfied" group suggests we ought to focus on meaning in life in student guidance in schools.

The person-centered approach allowed us to not only identify adolescents with homogeneous importance and satisfaction profiles across various life domains but also to examine the configurations within the profile from a holistic perspective. This strategy does not compete with the variable-centered approach, but plays a complementary role in 


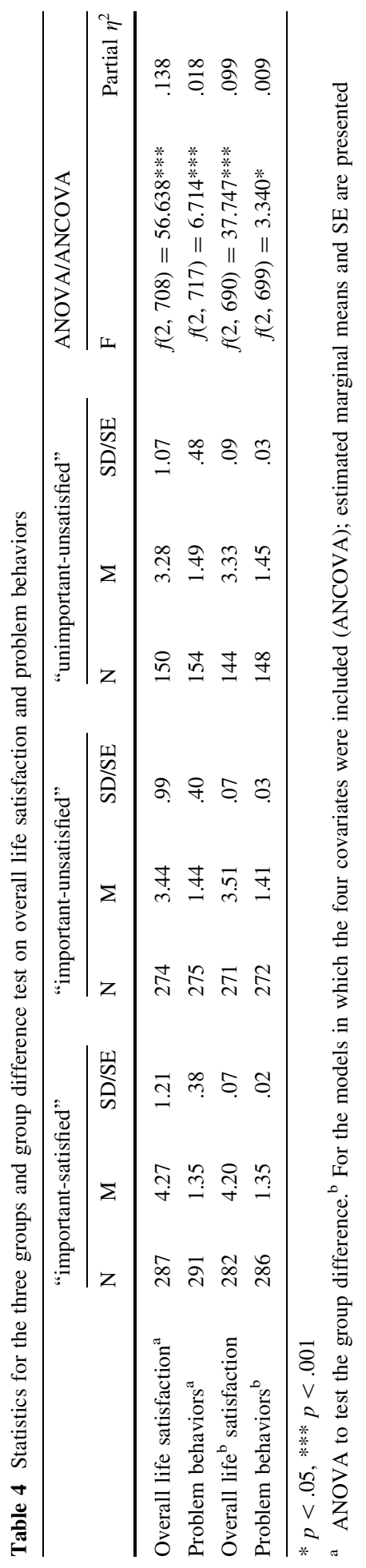


Table 5 ANCOVA result for the effect of three groups on overall life satisfaction after gender, level, living area, and health rating were also included as predictors

\begin{tabular}{|c|c|c|c|c|c|}
\hline $\begin{array}{l}\text { Dependent } \\
\text { variable }\end{array}$ & $\begin{array}{l}\text { Independent } \\
\text { variable }\end{array}$ & $d f$ & MS & $\mathrm{F}$ & Partial $\eta^{2}$ \\
\hline \multirow[t]{7}{*}{ Overall life satisfaction ${ }^{a}$} & Intercept & 1 & 796.597 & $678.794 * * *$ & .496 \\
\hline & 3 groups & 2 & 44.298 & $37.747 * * *$ & .099 \\
\hline & Gender & 1 & .002 & .002 & .000 \\
\hline & Level & 1 & 1.391 & 1.185 & .002 \\
\hline & Area & 1 & .919 & .783 & .001 \\
\hline & Health & 1 & 30.507 & $25.996 * * *$ & .036 \\
\hline & Error & 690 & 1.174 & & \\
\hline \multirow[t]{7}{*}{ Problem behaviors $^{\mathrm{b}}$} & Intercept & 1 & 186.612 & $1257.484 * * *$ & .643 \\
\hline & 3 groups & 2 & .496 & $3.340^{*}$ & .009 \\
\hline & Gender & 1 & 9.166 & $61.765 * * *$ & .081 \\
\hline & Level & 1 & .576 & $3.883^{*}$ & .006 \\
\hline & Area & 1 & .485 & 3.269 & .005 \\
\hline & Health & 1 & .834 & $5.619^{*}$ & .008 \\
\hline & Error & 699 & .148 & & \\
\hline
\end{tabular}

$* p<.05, * * * p<.001$

${ }^{\mathrm{a}} R^{2}=.173,{ }^{\mathrm{b}} R^{2}=.114$

providing more evidence on these research questions (Magnusson 2003). With a latent profile analysis, we were able to identify these groups of students and carry out further research to understand adolescents' sense of well-being.

\subsection{Relationships with Covariates}

With respect to the backgrounds of the students, we found that boys were more likely in the "unimportant-unsatisfied" group. In other words, boys tended to perceive life domains with a lack of concern. Most studies have suggested that girls are more depressed and more unsatisfied with life than are boys (e.g., Goldbeck et al. 2007). However, for certain domains (relationship with friends, school, and self), girls show more satisfaction than do boys (Huebner et al. 2004). Our finding revealed, from a different angle, that boys have a higher likelihood than girls of having an unconcerned attitude and of feeling unsatisfied with their well-being.

Goldbeck et al. (2007) noted that increased age brings increased dissatisfaction with certain life domains during adolescence. Huebner et al. (2004), on the other hand, found a weak relationship between age and life satisfaction. Whether there is an age difference in QoL during adolescence was not concluded. In the present study, high school students were more likely to be in the "unimportant-unsatisfied" group than were middle school students. A possible explanation could be that middle school students experience slightly less academic pressure than do high school students, who face more difficult and challenging school work (Wikipedia, n.d.). The finding based on only two categorical educational levels (middle and high schools) could not provide sufficient evidence to support the age difference; therefore, future research should be carried out using samples with a large range 
of age and preferably controlling confounding variables such as school learning environment.

Researchers have found statistically significant correlations between self-perceived health and overall life satisfaction (Zullig et al. 2006). Empirical evidence shows that selfrated health is one of the predictors of life satisfaction (Benyamini et al. 2004). In the present study, healthier students were less likely to be in the "important-unsatisfied" or "unimportant-unsatisfied" group, supporting the idea that healthier students are more likely to be satisfied with their lives. Moreover, when considering both perceived importance and satisfaction, less healthy students were unsatisfied with their lives, regardless of whether they perceived the domains to be important.

The relationships between the 3 latent groups and the overall life satisfaction criteria showed that there were no differences in overall life satisfaction between the "importantunsatisfied" and "unimportant-unsatisfied" groups. This finding seems to support the idea that considering domain importance does not improve the prediction of overall life satisfaction from domain satisfaction (Wu and Yao 2006a). Students who were unsatisfied with life had low overall life satisfaction, regardless of whether they considered the various life domains important or not.

Preventing problem behaviors, such as delinquency, violence, aggression, and skipping school in adolescence, is vitally important to the well-being of young people, and ultimately, to our society. Therefore, besides life satisfaction, we adopted the problem behavior rating as a negative well-being indicator and evaluated its relationships to the three groups of adolescents. We found that the "important-unsatisfied" and "unimportantunsatisfied" groups showed more problem behaviors than did the "important-satisfied" group. However, no difference was observed between the "important-unsatisfied" and "unimportant-unsatisfied" groups. This finding implies that perceived importance was not a factor in the relationship between importance-satisfaction profiles and the negative indicator of well-being. In a previous study, Wu and Yao (2006a) argued that importance does not play a moderating role in the prediction of life satisfaction from domain satisfaction. Some might argue that the domain satisfaction ratings are naturally more relevant to overall life satisfaction; it is reasonable that the moderator effects might not be significant. Therefore, using a well-being criterion other than overall life satisfaction would provide less controversial evidence. Our finding complements Wu and Yao's (2006a) findings and supports the idea that domain importance ratings play a trivial role in the prediction of well-being.

\subsection{Conclusions}

By using a different scale and a different sample, the present study provides added value to QoL studies that have evaluated both life domain importance and satisfaction ratings. In the past, researchers have used well-developed QoL scales in which the life domains are most relevant to their own samples or the items have been selected/revised over the years to fulfill the needs of their research. Thus, the QoL items usually reflect the life domains that are important to the particular samples. In the present study, we used the QOLPAV framework, developed based on a Centre for Health Promotion Model (CHP; Raphael et al. 1996), to cover physical being, psychological being, spiritual being, physical belonging, social belonging, community belonging, practical becoming, leisure becoming, and growth becoming. The scale items are comprehensive but not necessarily all important to the adolescents, thus making the contribution of importance ratings more prominent (Hsieh 2011). Moreover, although this scale has not been thoroughly studied in cross-cultural 
contexts, it has the potential for widespread use in assessing QoL for different adolescent populations (Zekovic and Renwick 2003) and reflecting individual differences. Therefore, this scale is particularly suited for the investigation of various types of adolescents based on domain importance and satisfaction ratings.

Another significant contribution of the present study is the target sample of this study. Instead of focusing on the elderly population or college students, we were more concerned with QoL for youth. This study provides a different emphasis, focusing on people who are in a life stage in which significant physical and mental changes are occurring. Identifying distinct groups based on subjective life satisfaction and perceived importance will shed light on the understanding of adolescents' perception of QoL and further improve their well-being.

There has been great debate about whether overall QoL indices should take importance weightings into account. Specifically, multiplicative methods have been criticized as lacking proper psychometric foundations. However, not only have multiplicative methods been abolished, but so has the concept of importance. Our study reveals two-sided implications. First, although indirect, the evidence from our study supports the findings of $\mathrm{Wu}$ and Yao (2006a). We deem that where the measures that predict well-being among youth are concerned, satisfaction ratings are sufficient for this purpose.

The argument of why we should (or not) weight, or how we should weight QoL by incorporating importance into satisfaction scores, was not the focus of the present study. However, Wallander et al. (2001) noted that importance weights are crucial elements of QOL measurement. Trauer and Mackinnon (2001) also argued that the importance of a particular life aspect in a satisfaction scale has explanatory value and should not be neglected. On the other side of our two-sided results, we agree that importance provides valuable information and advocate that perceived importance plays a role that should not be neglected in research on adolescents' well-being. We identified one type of adolescents who have less positive attitudes toward the importance of and satisfaction with a variety of life domains, thus providing important implications for educators and parents. Lyubomirsky et al. (2005) noted that cognitive and motivational strategies influence one's attitudes toward life and happiness. Thus, personal education and intervention activities that cultivate adolescents' positive feelings, cognitions, and behaviors should be implemented in the school and in the community to enhance well-being among youth (Sin and Lyubomirsky 2009).

\subsection{Limitations and Future Studies}

Excluding younger samples is one of the limitations of the present study. A possible developmental trend in the latent profiles might exist; however, no study, including the present one, has investigated longitudinal changes in general QoL among adolescents and children. In addition, the stability of the latent profiles of perceived importance of and satisfaction with life domains was not addressed in this study due to the restriction of a cross-sectional design. Therefore, it is important to include younger samples and conduct the survey using a longitudinal design in the future.

Another major limitation of this study is that we adopted only quantitative methods to explore the adolescent groups that show similar rating profiles of importance and satisfaction in a QoL scale. The psychological process was not postulated in terms of how the value of importance is considered by an individual when rating his or her satisfaction with a particular life domain. For example, the psychological process underlying the ratings of the "unimportant-unsatisfied" group needs to be examined using an alternative technique 
(e.g., qualitative). It is worthwhile to know why the adolescents with unconcerned attitudes toward life domains selected "unsatisfied," not a neutral rating as depicted in the range-ofaffect hypothesis.

There are several other reasons that the investigation of life domain importance and satisfaction should continue. Although the present study did not intend to discuss the issue of incorporating importance weighting in the overall $\mathrm{QoL}$ index, there is still room to investigate the position importance weighting should have in the larger puzzle. Additionally, some studies have examined the latent construct (or factors) of life satisfaction and QoL (Hsieh 2008; Wu et al. 2009; Wu and Yao 2007b). However, to our knowledge, no one has investigated importance and satisfaction factors simultaneously (Russell and Hubley 2005). Although our study evaluated the importance and satisfaction constructs simultaneously to analyze latent profiles, still more detailed evaluations of these constructs are needed, especially as applied to a greater variety of populations.

Acknowledgments This research is part of the "Taiwan Student Physical and Mental Development Study" funded by the ROC National Academy of Educational Research (NAER-95-24-A-2-01-00-2-14). Additional support came from the ROC National Science Council through two projects: "The Net and Taiwan Adolescents' Physical and Mental Development" (NSC 97-2631-S-009-001) and "Internet Use and Well-being of Taiwan Students: Longitudinal Analysis and the Extension of Fact Bank" (NSC-100-2631-S009-001 and NSC-101-2631-S-009-001).

\section{References}

Akaike, H. (1987). Factor analysis and AIC. Psychometrika, 52, 317-332. doi:10.1007/BF02294359.

Benyamini, Y., Leventhal, H., \& Leventhal, E. A. (2004). Self-rated oral health as an independent predictor of self-rated general health, self-esteem and life satisfaction. Social Science and Medicine, 59, 1109-1116. doi:10.1016/j.socscimed.2003.12.021.

Bradford, R., Rutherford, D. L., \& John, A. (2002). Quality of life in young people: Ratings and factor structure of the quality of life profile-adolescent version. Journal of Adolescence, 25, 261-274. doi: $10.1006 /$ yjado/469.

Chen, S. K., Hwang, F. M., \& Lin S.S.J. (2011) Satisfaction ratings of QOLPAV: Psychometric properties based on the graded response model. Social Indicators Research, 1-17. doi: 10.1007/s11205-011-9935-1.

Diener, E., Emmons, R. A., Larsen, R. J., \& Griffin, S. (1985). The satisfaction with life scale. Journal of Personality Assessment, 49, 71-75.

Goldbeck, L., Schmitz, T. G., Besier, T., Herschbach, P., \& Henrich, G. (2007). Life satisfaction decreases during adolescence. Quality of Life Research, 16, 969-979. doi:10.1007/s11136-007-9205-5.

Herman, K. C., Ostrander, R., Walkup, J. T., Silva, S. G., \& March, J. S. (2007). Empirically derived subtypes of adolescent depression: Latent profile analysis of co-occurring symptoms in the treatment for adolescents with depression study (TADS). Journal of Consulting and Clinical Psychology, 75, 716-728. doi:10.1037/0022-006X.75.5.716.

Ho, M. Y., Cheung, F. M., \& Cheung, S. F. (2010). The role of meaning in life and optimism in promoting well-being. Personality and Individual Differences, 48, 658-663. doi:10.1016/j.paid.2010.01.008.

Hsieh, C. (2003). Counting importance: The case of life satisfaction and relative domain importance. Social Indicators Research, 61, 227-240.

Hsieh, C. (2008). The relative importance of health. Social Indicators Research, 87, 127-137. doi: $10.1007 / \mathrm{s} 11205-007-9162-y$.

Hsieh, C. (2011). Issues in evaluating importance weighting in quality of life measures. Social Indicators Research. Retrieved from http://link.springer.com/article/10.1007/s11205-011-9951-1/fulltext.html

Hsieh, C. (2012a). Importance is not unimportant: The role of importance weighting in QOL measures. Social Indicators Research, 109, 267-278. doi:10.1007/s11205-011-9900-z.

Hsieh, C. (2012b). Should we give up domain importance weighting in QoL measures? Social Indicators Research, 108, 99-109. doi:10.1007/s11205-011-9868-8.

Huebner, E. S., Valois, R. F., Suldo, S. M., Smith, L. C., McKnight, C. G., Seligson, J. L., et al. (2004). Perceived quality of life: A neglected component of adolescent health assessment and intervention. Journal of Adolescent Health, 34, 270-278. doi:10.1016/S1054-139X(03)00285-4. 
Lo, Y., Mendell, N., \& Rubin, D. (2001). Testing the number of components in a normal mixture. Biometrika, 88, 767-778. doi:10.1093/biomet/88.3.767.

Locke, E. A. (1969). What is job satisfaction? Organizational Behavior and Human Performance, 4, 309-336. doi:10.1016/0030-5073(69)90013-0.

Lyubomirsky, S., King, L., \& Diener, E. (2005). The benefits of frequent positive affect: Does happiness lead to success? Psychological Bulletin, 131, 803-855. doi:10.1037/0033-2909.131.6.803.

Magidson, J., \& Vermunt, J. K. (2004). Latent class models. In D. Kaplan (Ed.), Handbook of quantitative methodology for the social sciences (pp. 345-368). Newbury Park, CA: Sage Publications. doi: 10.4135/9781412986311.n10.

Magnusson, D. (2003). The person approach: Concepts, measurement models, and research strategy. New Directions for Child and Adolescent Development, 2003, 3-23. doi:10.1002/cd.79.

Magnusson, D., \& Cairns, R. B. (1996). Developmental science: Toward a unified framework. In R. B. Cairns \& G. H. Elder Jr (Eds.), Developmental science. Cambridge studies in social and emotional development (pp. 7-30). New York: Cambridge University Press.

Meuleners, L. B., \& Lee, A. H. (2005). Quality of life profile-adolescents: Assessing the relationship of covariates to scale scores using structural equation modeling. Quality of Life Research, 14, 1057-1063. doi: 10.1007/s11136-004-2573-1.

Meuleners, L. B., Lee, A. H., Binns, C. W., \& Lower, A. (2003). Quality of life for adolescents: Assessing measurement properties using structural equation modeling. Quality of Life Research, 12, 283-290.

Muthén, B. O. (2001). Latent variable mixture modeling. In G. A. Marcoulides \& R. E. Schumacker (Eds.), New developments and techniques in structural equation modeling (pp. 1-33). Hillsdale, NJ: Lawrence Erlbaum.

Muthén, L. K., \& Muthén, B. O. (2010). Mplus User's Guide (6th ed.). Los Angeles, CA: Muthén \& Muthén.

Raphael, D., Rukholm, E. B., Hill-Bailey, P., \& Donato, E. (1996). The quality of life profile-adolescent version: Background, description, and initial validation. Journal of Adolescent Health, 19, 366-375. doi:10.1016/S1054-139X(96)00080-8.

Russell, L. B., \& Hubley, A. M. (2005). Importance ratings and weighting: Old concerns and new perspectives. International Journal of Testing, 5, 105-130. doi:10.1207/s15327574ijt0502_2.

Russell, L. B., Hubley, A. M., Palepu, A., \& Zumbo, B. D. (2006). Does weighting capture what's important? Revisiting subjective importance weighting with a quality of life measure. Social Indicators Research, 75, 141-167. doi:10.1007/s11205-004-2528-5.

Schwartz, G. (1978). Estimating the dimension of a model. The Annals of Statistics, 6, 461-464.

Sclove, S. L. (1987). Application of model-selection criteria to some problems in multivariate analysis. Psychometrika, 52, 333-343. doi:10.1007/BF02294360.

Sin, N. L., \& Lyubomirsky, S. (2009). Enhancing well-being and alleviating depressive symptoms with positive psychology interventions: A practice-friendly meta-analysis. Journal of Clinical Psychology, 65, 467-487. doi:10.1002/jclp.20593.

Trauer, T., \& Mackinnon, A. (2001). Why are we weighting? The role of importance ratings in quality of life measurement. Quality of Life Research, 10, 579-585.

Tuominen-Soini, H., Salmela-Aro, K., \& Niemivirta, M. (2008). Achievement goal orientations and subjective well-being: A person-centred analysis. Learning and Instruction, 18, 251-266. doi:10.1016/ j.learninstruc.2007.05.003.

Wallander, J. L., Schmitt, M., \& Koot, H. M. (2001). Quality of life measurement in children and adolescents: Issues, instruments, and applications. Journal of Clinical Psychology, 57, 571-585. doi: 10.1002/jclp.1029.

Wekipedia. (n.d.). Educatoin in Taiwan. Retrieved Nov. 8, 2012, from http://en.wikipedia.org/wiki/ Education_in_Taiwan

Wu, C. H. (2008a). Examining the appropriateness of importance weighting on satisfaction score from range-of-affect hypothesis: Hierarchical linear modeling for within-subject data. Social Indicators Research, 86, 101-111. doi:10.1007/s11205-007-9103-9.

Wu, C. H. (2008b). Can we weight satisfaction score with importance ranks across life domains? Social Indicators Research, 86, 469-480. doi:10.1007/s11205-007-9180-9.

Wu, C. H., Chen, L. H., \& Tsai, Y. M. (2009). Investigating importance weighting of satisfaction scores from a formative model with partial least squares analysis. Social Indicators Research, 90, 351-363. doi:10.1007/s11205-008-9264-1.

Wu, C. H., \& Yao, G. (2006a). Do we need to weight item satisfaction by item importance? A perspective from Locke's range-of-affect hypothesis. Social Indicators Research, 79, 485-502. doi:10.1007/ s11205-005-5666-5. 
Wu, C. H., \& Yao, G. (2006b). Do we need to weight satisfaction scores with importance ratings in measuring quality of life? Social Indicators Research, 78, 305-326. doi:10.1007/s11205-005-0212-z.

Wu, C. H., \& Yao, G. (2007a). Importance has been considered in satisfaction evaluation: An experimental examination of Locke's range-of-affect hypothesis. Social Indicators Research, 81, 521-541. doi: 10.1007/s11205-006-0021-z.

Wu, C. H., \& Yao, G. (2007b). Examining the relationship between global and domain measures of quality of life by three factor structure models. Social Indicators Research, 84, 189-202. doi: 10.1007/s11205-006-9082-2.

Zekovic, B., \& Renwick, R. (2003). Quality of life for children and adolescents with developmental disabilities: Review of conceptual and methodological issues relevant to public policy. Disability and Society, 18, 19-34.

Zullig, K. J., Ward, R. M., \& Horn, T. (2006). The association between perceived spirituality, religiosity, and life satisfaction: The mediating role of self-rated health. Social Indicators Research, 79, 255-274. doi:10.1007/s11205-005-4127-5. 